\title{
Continuous Decoupling of Dynamically Expanding Systems
}

\author{
Jörn Knoll ${ }^{1}$ \\ Gesellschaft für Schwerionenforschung \\ Planckstr. 1 \\ 64291 Darmstadt
}

\begin{abstract}
The question of decoupling and freeze-out is reinvestigated and analysed in terms of transparent semi-classical decoupling formulae, which provide a smooth decoupling in time both, for single and two particle inclusive spectra. They generalise frequently employed instantaneous freeze-out procedures and provide simple relations between the damping width and the duration of the decoupling process. The implications on physical phenomena arising from the expansion and decay dynamics of the highly compressed hadronic matter generated in high energy nuclear collisions are discussed.
\end{abstract}

Key words: decoupling; freeze-out; phase transitions;

PACS: 25.75.-q, 24.10.Nz, 24.10.Eq

\section{Introduction}

Dynamically expanding systems such as those created in high energy nuclear collisions or the early universe pass various stages, where different degrees of freedom are relevant and thus different dynamical concepts are appropriate. In the context of nuclear collisions, cf. [1], one envisages a phase, where at high densities partonic degrees of freedom prevail in form of a quark gluon plasma (QGP) that then during the expansion converts to a dense hadronic medium. Subsequently the chemical components (i.e. the abundances of the different hadrons) decouple and finally the system kinetically freezes out releasing the particles that reach the detectors. The early universe passed through various transition stages like the electro-weak transition, or the neutrino or photon decoupling, the latter leading to the micro-wave background radiation.

$\overline{1}$ e-mail:j.knoll@gsi.de 
All such transitions share that they proceed during quite some time. The transition can be accompanied by a strong change in the entropy density and thus releases quite some amount of latent heat, as e.g. during the QGP to hadron phase transition. Thereby the transition dynamics requires adjustments of the structural and thermodynamic properties of the system.

Continuous transition processes can properly be described in the context of coupled transport equations, like the Boltzmann or e.g. chemical rate equations, as e.g. presented in [2-7]. Still, sudden transition schemes are frequently employed, be it in the context of macro-dynamical descriptions or simply for a survey analyses of a wide body of the nuclear collision data. These concern coalescence pictures, which combine nucleons to composite nuclei at freezeout [8] or coalesce quarks to hadrons in the deconfinement-confinement transition, e.g. [9]. Such recipes do not only bypass the transition dynamics. In many cases they violate general principles as detailed balance, unitarity, conservation laws or entropy requirements $[6,7]$. Also the general freeze-out of the diluting system is mostly treated as a sudden transition happening at a suitably chosen three dimensional freeze-out hyper-surface in space-time [10]. Commonly one then defines a proper transition condition in terms of a transition temperature or transition density. In particular the latter freeze-out picture is widely used to analyse nuclear collision data in terms of thermal models. The achieved fits in temperature, chemical potentials and parametrised flow effects to the observed particle abundances and kinetic spectra are then frequently used as measured data that are taken as clues on the physics of the collision dynamics, cf. Refs. [11-14].

In this note we reinvestigate transition and decoupling processes under the perspective that these processes proceed during some considerable time span. Here we concentrate on the physics aspects of the continuous decoupling processes and shall defer the conceptual derivation, based on a final state interaction picture within the non-equilibrium formulation of quantum many-body theory as given by Kadanoff and Baym (KB) [15], to a forthcoming paper [16].

Starting from an exact microscopic formulation in terms of distorted waves [16-18] transparent semi-classical relations are obtained that describe a continuous decoupling or freeze-out of strongly interaction particles. These formulae generalise the instantaneous freeze-out concepts, like the Cooper-Frye formulae [10], to finite decoupling time effects. Thereby the drain of energymomentum and conserved currents from the source needs to be considered in order to come to an overall conserving scheme. The concept offers a direct generalisation to two-particle coincidence rates known as the Hanburry-BrownTwiss (HBT) effect [19] using distorted waves [20,21].

Earlier efforts [22-26] concentrated on introducing a finite depth of the decoupling layer, across which a fluid phase is coupled to a gas of frozen-out particles. Those attempts simply parametrised the finite decoupling, rather than 
inferring it microscopically [27] from the underlying decoupling processes, as will be considered here. The microscopic treatment permits to account for the individual properties of the particles, which e.g. leads to specific conclusions on the fate of short lived resonances during freeze-out. The latter is closely related to the generic balance between the local creation and the subsequent absorption during escape, which is finally responsible for an erosion of the memory on structural effects of the source.

In the discussion section various decoupling phenomena, such as the chemical and thermal freeze-out, but also implications on phase transitions in nuclear collisions are reinspected under the particular perspective, that transition processes proceed during a considerable time span, across which the bulk properties of the expanding system may significantly change.

We use the convention $\hbar=c=1$ and formulate all relations for relativistic scalar bosons, for which standard covariant notation is used.

\section{Decoupling rates}

The invariant single-particle detector rate of a particle $a$ (scalar boson) can be formulated as $[16-18] 2$

$$
\begin{aligned}
2 p_{A}^{0} \frac{d N_{a}\left(p_{A}\right)}{d^{3} p_{A}} & =\int \frac{d^{4} x d^{4} y}{(2 \pi)^{3}} \underbrace{\left\langle J_{a}^{\dagger}(x) J_{a}(y)\right\rangle_{\text {irred. }}}_{\Pi_{\text {gain }}(x, y)} \psi_{\vec{p}_{A}}^{(-) \dagger}(y) \psi_{\vec{p}_{A}}^{(-)}(x) \\
& =\frac{1}{(2 \pi)^{3}}\left\langle\psi_{\vec{p}_{A}}^{(-) \dagger}\left|\Pi_{a}^{\text {gain }}\right| \psi_{\vec{p}_{A}}^{(-)}\right\rangle
\end{aligned}
$$

Here the current-current correlation function $\Pi^{\text {gain }}$ encodes the property of the source, while the distorted single particle wave functions $\psi_{\vec{p}_{A}}$ describe the final state the propagation of the observed particle. This includes optical deflection and absorption effects through the real and imaginary parts of the retarded polarisation function $\Pi_{a}^{\mathrm{R}}\left(x, x^{\prime}\right)=i \Theta\left(t-t^{\prime}\right)\left\langle\left[J_{a}^{\dagger}(x), J_{a}\left(x^{\prime}\right)\right]\right\rangle_{\text {irred. }}$. Expression (1) is rigorous, provided one knows the exact one-particle irreducible currentcurrent correlation function and the corresponding exact final-state distorted wave $\psi_{\vec{p}_{A}}^{(-)}$. The latter results from the outgoing scattering solution of the KleinGordon equation governed by $\Pi_{a}^{\mathrm{R}}\left(x, x^{\prime}\right)$ with corresponding asymptotic boundary condition $[28,29]$. The technical and further conceptual developments will be the subject of a forthcoming paper [16].

$\overline{2}$ The current field operator $J_{a}(x)$ used in (1) formally results from the functional variation of the interaction Lagrangian with respect to the field operator $\phi_{a}(x)$ of particle $a$ as $J_{a}(x)=\delta \mathcal{L}^{\text {int }}(\phi) / \delta \phi_{a}^{\dagger}(x)$. 


\subsection{Semi-classical decoupling rates}

The semi-classical approximation provides a physically intuitive formulation in terms of classical paths. It either amounts to consider JWKB like approximations for the distorted waves, leading to a covariant Hamilton-Jacobi problem for the classical action [30], or equivalently to perform the first order gradient approximation to the Kadanoff-Baym (KB) equations [15,31,32]. In the small damping-width limit the approximate expressions for both cases are then unique and constructed through the bundle of covariant classical paths determined by the real part of $\Pi^{\mathrm{R}}$. The paths obey the on-shell constraint, while the imaginary damping part of the classical action is treated perturbatively. Then each real path accounts for the entire local spectral width.

In the following we will discuss two decoupling schemes: a) the local decoupling rate that can be even off-shell and b) the detector yield. For case a) the gain term, $\Pi^{\text {gain }}(x, p) A(x, p)$, of the gradient expanded KB equation [15,31-34], where $A$ is the spectral function, suggests the following local decoupling rate

$$
\frac{d N(x, p)}{d^{4} p d t d^{3} x}=\frac{1}{(2 \pi)^{4}} \Pi^{\text {gain }}(x, p) A_{a}(x, p) \mathcal{P}_{\text {escape }}(x, p)
$$

Here $\mathcal{P}_{\text {escape }}(x, p)$ captures the probability that particles, created or scattered at space-time point $x$ into a momentum $p$, can escape to infinity without further being absorbed by the loss part of the collision term. This formulation thus restricts the emission zone to the layer of the last interaction. Semiclassically in the small width limit $\mathcal{P}$ is given as

$$
\begin{aligned}
\mathcal{P}_{\text {escape }}(x, p) & =\mathrm{e}^{-\chi(x, p)}, \quad \text { where } \\
\chi(x, p) & =\int_{(x, \vec{p})}^{\infty} \Gamma\left(x^{\prime}, p^{\prime}\right) d t^{\prime}, \quad \text { and } \Gamma\left(x^{\prime}, p^{\prime}\right)=-\operatorname{Im} \Pi^{\mathrm{R}}\left(x^{\prime}, p^{\prime}\right) / p_{0}^{\prime},
\end{aligned}
$$

where the time integration is taken along the classical escape path starting at $(x, \vec{p})$. The exponent $\chi$ is also known as optical depth. At this point one may be tempted to assume that the local decoupling rate (3) together with (4) could also apply to the broad spectral-width case, upon generalising the classical paths to appropriate off-mass shell paths. As yet a proof in compliance the overall conservation laws could so far not be given ${ }^{3}$. We'll nonetheless keep this intuitive formulation, which cares about both facets of damping, the attenuation of the flux as well as the corresponding spectral-width dynamics, implicitly hoping that this form can be applied under much broader circumstances as used for its derivation.

3 The occurrence of a back-flow term [31] objects to an exact formulation in terms of classical paths. A special approximation though was discussed in [31,33,34]. 
Under the small width assumption that the spectral strength $A(x, p)$ will be guided via the on-shell path, starting from $(x, \vec{p})$ towards an on-shell detector momentum $\vec{p}_{A}$, leads to the following detector yield

$$
\begin{aligned}
\frac{d N_{a}\left(p_{A}\right)}{d^{3} p_{A}} & =\int \frac{d^{4} x d^{4} p}{(2 \pi)^{4}} \Pi_{a}^{\text {gain }} A_{a} \mathcal{P}_{\text {escape }} \delta^{3}\left(\vec{p}_{A}-\vec{p}_{A}(x, \vec{p})\right) \\
& =\int \frac{d^{4} x d^{4} p}{(2 \pi)^{4}} \Pi_{a}^{\text {gain }} A_{a} \mathcal{P}_{\text {escape }}\left(\frac{\partial \vec{p}_{A}}{\partial \vec{p}}\right)^{-1} \delta^{3}\left(\vec{p}-\vec{p}\left(x, \vec{p}_{A}\right)\right)
\end{aligned}
$$

Here $\vec{p}_{A}(x, \vec{p})$ denotes the corresponding mapping of the local momentum $\vec{p}$ to the detector momentum. The inverse mapping $\vec{p}\left(x, \vec{p}_{A}\right)$ may neither be unique, nor even existing for some values of $x$ (classical shadow regions which don't contribute). The corresponding Jacobi determinant accounts for the focussing or defocusing of the classical paths due to deflections by the optical potential resulting from Re $\Pi^{\mathrm{R}}$. Owing to Liouville's theorem this Jacobian agrees with the van Vleck determinant [35] appearing as the pre-exponential factor in the JWKB ansatz for the distorted waves in (1).

In the narrow width limit the above rates and yields naturally merges known continuous freeze-out pictures [22-26] of free gas kinetics, here however used microscopically with due account of the individual source terms and optical potential. The formulae encompass the completely opaque (i.e. strong interaction) limit as well as that for weakly interacting i.e. penetrating probes. For the latter case final-state distortion effects are negligible and one exactly recovers the known Golden-Rule result

$$
\begin{aligned}
\frac{d N_{a}(x, p)}{d^{4} p d^{4} x}= & \frac{1}{(2 \pi)^{4}} \Pi_{a}^{\mathrm{gain}}(x, p) A_{a}^{\mathrm{vac}}(p) \quad \text { (for weakly interacting probes) } \\
& \text { with } \quad A_{a}^{\mathrm{vac}}(p)=2 \pi \delta\left(p^{2}-m_{a}^{2}\right)
\end{aligned}
$$

here formulated in terms of the current-current correlator or polarisation function, which still can account for non-perturbative multiple collision contributions from the source [36].

\section{Strong decoupling and freeze-out}

If one wants to describe the dynamics in terms of a two component scenario [22-26] with an interacting source and a decoupled (frozen-out) component, the above local decoupling rate (3) has to be supplemented by a corresponding description of the depopulation of the source. It causes a drain in particle number and energy and a recoil momentum. In fluid dynamical descriptions this leads to the following loss terms in the corresponding fluid cells 


$$
\begin{aligned}
\partial_{\mu} j_{\alpha, \text { fluid }}^{\mu}(x) & =-\sum_{a} e_{a \alpha} \int d^{4} p \frac{d N_{a}(x, p)}{d^{4} x d^{4} p} \\
\partial_{\mu} T_{\text {fluid }}^{\mu \nu} & =-\sum_{a} \int d^{4} p p^{\nu} \frac{d N_{a}(x, p)}{d^{4} x d^{4} p} .
\end{aligned}
$$

These transfer rates result from the dissipative part of the gradient expanded $\mathrm{KB}$ equations, namely upon weighting the loss term with the charge $e_{a \alpha}$ of particle $a$ or with $p^{\nu}$, respectively. Here $\alpha$ labels a conserved charge. These recoupling terms have forms similar to those used in multi-fluid models, cf. [2225,37-42]. Here, however, the decoupling of individual particles is addressed with their microscopic properties rather than an "anonymous" decoupling between two fluids. A complete treatment of the decoupling rate (3) together with the fluid drain terms (8) then provides an overall conserving scheme. Such recouplings lead to a gradual fading of the fluid phase, upon creating the freely streaming particle phase. Due to the a posteriori nature of the decoupling equations (3), since they involve the knowledge on the future, a solution of this coupled dynamics may only be obtained iteratively. As, however, pointed out in [27] such a two-component picture may not be quite consistent, since particles in both components are subjected to the same interaction dynamics and the tagging of the frozen-out particles is solely a matter of probabilities.

\section{Thermal source limit}

In thermal equilibrium the source function $\Pi^{\text {gain }}$ can directly be expressed as

$$
\Pi_{a}^{\text {gain }}(x, p)=-2 f_{\mathrm{th}}\left(x, p^{0}\right) \operatorname{Im}_{\Pi_{a}^{\mathrm{R}}}^{\mathrm{R}}(x, p)=f_{\mathrm{th}}\left(x, p^{0}\right) 2 p^{0} \Gamma_{a}(x, p),
$$

where $\Gamma_{a}(x, p)$ is the local damping width of particle $a$. This property leads to quite some compensating effect, which is frame independent. Namely, for large source extensions the integral over the $\Gamma$-dependent damping factors in (5), which define the visibility probability $P_{t}$, integrated along any path leading from the opaque interior to the outside, equates to unity

$$
\int_{-\infty}^{\infty} d t \underbrace{\Gamma(t) \mathrm{e}^{-\chi(t)}}_{=P_{t}(t)}=1, \quad \text { where } \chi(t)=\int_{t}^{\infty} d t^{\prime} \Gamma\left(t^{\prime}\right) .
$$

This compensation is independent of the structural details of $\Gamma$ and of the classical paths leading to the detector, along which the decoupled particles are accumulated. It is valid as long as space-time changes in $\Pi$ or $\Gamma$ are sufficiently smooth as to permit the underlying semi-classical considerations. In case of sharp changes in $\Pi$ the occurrence of reflection and diffraction effects [43] may partially spoil the picture. When rates drop smoothly in time, the visibility probability $P_{t}(t)$ achieves its maximum at 


$$
\left[\dot{\Gamma}(t)+\Gamma^{2}(t)\right]_{t_{\max }}=0, \quad \text { where } \quad P_{t}\left(t_{\max }\right) \approx \Gamma\left(t_{\max }\right) / \mathrm{e}
$$

Here the dot denotes the time derivative along the corresponding classical path. The corresponding decoupling duration $\Delta t_{\text {dec }}$ approximately follows from the normalisation of the visibility function $P_{t}$ to

$$
\Delta t_{\mathrm{dec}} \approx \frac{1}{P_{t}\left(t_{\max }\right)} \approx \frac{\mathrm{e}}{\Gamma\left(t_{\max }\right)}
$$

This defines a kind of decoupling uncertainty relation $\Gamma\left(t_{\max }\right) \Delta t_{\mathrm{dec}} \approx \mathrm{e}$.

Collecting all rates along the bundle of classical paths leading to the detector according to (5) provides

$$
\begin{array}{r}
(2 \pi)^{4} \frac{d N_{a}\left(\vec{p}_{A}\right)}{d^{3} p_{A}}=\int d^{4} x 2 p^{0} d p^{0} f_{\mathrm{th}}\left(x, p^{0}\right)\left[\frac{\partial \vec{p}}{\partial \vec{p}_{A}} A_{a}(x, p) \Gamma_{a}(x, p) \mathrm{e}^{-\chi(x, p)}\right] \\
=\int \underbrace{d^{3} \sigma_{\mu} d x^{\mu}}_{d^{4} x} 2 p^{0} d p^{0} f_{\mathrm{th}}\left(x, p^{0}\right)\left[\frac{\partial \vec{p}}{\partial \vec{p}_{A}} A_{a}(x, p) \Gamma_{a}(x, p) \mathrm{e}^{-\int_{t}^{\infty} d t^{\prime} \Gamma_{a}\left(x^{\prime}, p\right)}\right],
\end{array}
$$

where the square bracket expressions [...] are to be taken at the corresponding classical three-momentum $\vec{p}\left(x, \vec{p}_{A}\right)$ of the local detector orbit. This rate generalises the Cooper-Frye formula [10] to a smooth decoupling situation dictated by the properties of the damping rate during the decoupling process. Similar continuous decoupling concepts, though based on the Boltzmann equation, were used in cosmology already for quite some time, see e.g. [44] and for a recent text book in this context [45], Chapt. 9. In (14) the space-time integration is expressed in terms of 3 dimensional hyper-surfaces layers $\sigma$ in the sense of curved coordinates. For each momentum each surface $\sigma$ can e.g. be defined as surfaces of constant $\Gamma(x, p)$, while $d x^{\mu}$ defines the world line direction towards the detector.

In the opaque limit of the source and as long as the matter properties such as the temperature do not significantly change across the decoupling process the integral property (10) leads to an improved Cooper-Frye picture

$(2 \pi)^{4} \frac{d N_{a}\left(\vec{p}_{A}\right)}{d^{3} p_{A}} \underset{\mathrm{CF}}{\longrightarrow} \int_{\sigma_{\mathrm{fo}}\left(\vec{p}_{A}\right)} d^{3} \sigma_{\mu} p^{\mu} d p^{0}\left[\frac{\partial \vec{p}}{\partial \vec{p}_{A}} f_{\mathrm{th}}\left(x, p^{0}\right) A_{a}^{\mathrm{fo}}(x, p)\right]_{\vec{p}\left(x, \vec{p}_{A}\right)}$

with freeze-out hypersurface $\sigma_{\mathrm{fo}}\left(\vec{p}_{A}\right)$. As a special ingredient this result is compatible with Planck's radiation law for static (i.e. spatial) surfaces, which the Cooper-Frye formula is not. The tiny but essential difference is that the freeze-out surface does depend on the detector momentum $\vec{p}_{A}[27,46] \square$. Thus

$\overline{4}$ Earlier attempts [24,47-49] tried to heuristically rescue this by a $\Theta$-function factor which is only non-zero for those particle momenta which permit the particles to leave 
viewing e.g. the collision zone from different sides leads to different freezeout zones. Therefore the mere assumption underlying the previous freeze-out pictures, that all particles touching a globally defined freeze-out hyper-surface do freeze out, is simply inappropriate especially for spatial surfaces. In order to recover a conserving scheme, though, the corresponding partial particle losses have to be accounted for by an appropriate surface recoupling term for the source fluid, the latter to be derived from the drain rates (8). The Cooper-Frye-Planck formula (15) is written as to include the spectral function $A_{a}^{\text {fo }}(x, p)$ of the released particle right after freeze-out, which may be used energy differentially across the spectral width. For unstable particles, like e.g. vector mesons [50], it may then serve as an input for the subsequent decay e.g. competitively into dileptons and hadrons.

The shining hyper-surface $\sigma_{\mathrm{fo}}\left(\vec{p}_{A}\right)$ in (15) can be determined for each observed momentum $\vec{p}_{A}$ from the space-time points of highest brilliance as given by (11) to

$$
\left[p ^ { \mu } \partial _ { \mu } \Gamma \left(x((t), p)+p^{0} \Gamma^{2}(x((t), p)]_{x \in \sigma_{\mathrm{fo}}\left(\vec{p}_{A}\right)}=0 .\right.\right.
$$

This provides a microscopic definition of a freeze-out criterion. A more general determination of the freeze-out surface, which refers to specific observables, is given in Eq. (28) in Sect. 5.

\section{Two particle final state correlations}

In straight generalisation of the microscopic definition of the freeze-out source (1) the two-particle source is given by the gain component of a double currentcurrent correlation function of the two observed particles $a$ and $b$ [51]

$$
S_{a b}\left(x_{a}, y_{a} ; x_{b}, y_{b}\right)=\left\langle J_{a}^{\dagger}\left(x_{a}^{-}\right) J_{a}\left(y_{a}^{+}\right) J_{b}^{\dagger}\left(x_{b}^{-}\right) J_{b}\left(y_{b}^{+}\right)\right\rangle
$$

with four-coordinates $x_{a}$ etc. Accounting for the complete final state interaction (FSI) of both particles the coincidence yield expected at two detectors with momenta $p_{A}$ and $p_{B}$ is then obtained by $[17,20,21]$

$$
\begin{aligned}
I_{a b}\left(\vec{p}_{A}, \vec{p}_{B}\right) & =\int d^{4} x_{a} d^{4} y_{a} d^{4} x_{b} d^{4} y_{b} \Psi_{\vec{p}_{A} \vec{p}_{B}}^{(-) \dagger}\left(y_{a}, y_{b}\right) S_{a b}\left(x_{a}, y_{a} ; x_{b}, y_{b}\right) \Psi_{\vec{p}_{A} \vec{p}_{B}}^{(-)}\left(x_{a}, x_{b}\right) \\
& =\left\langle\Psi_{\vec{p}_{A} \vec{p}_{B}}^{(-) \dagger}\left|S_{a b}\right| \Psi_{\vec{p}_{A} \vec{p}_{B}}^{(-)}\right\rangle .
\end{aligned}
$$

For details we refer to the review papers $[51,52]$ and the historical perspectives $[53,54]$.

the collision zone. This method lead to problems with respect to conservation laws. 
In general the complete final state two-body waves $\Psi$ require a full scale three body problem to be solved. In this note we rather concentrate on the simplifying case, where the mutual interaction between the observed pair is negligible. Then the two terms in the (anti-)symmetric two particle wave functions still factorise and the result for the correlated yield over the single yields can be cast into a form

$$
C\left(p_{A}, p_{B}\right)=1 \pm\left[\frac{\left|\left\langle\psi_{p_{A}}^{\dagger}\left|\Pi^{\text {gain }}\right| \psi_{p_{B}}\right\rangle\right|^{2}}{\left|\left\langle\psi_{p_{A}}^{\dagger}\left|\Pi^{\text {gain }}\right| \psi_{p_{A}}\right\rangle\left\langle\psi_{p_{B}}^{\dagger}\left|\Pi^{\text {gain }}\right| \psi_{p_{B}}\right\rangle\right|}\right]_{p_{A}^{2}=p_{B}^{2}=m^{2}}
$$

where now the single particle distorted waves $\psi_{p_{A}}$ etc. enter. Here the matrix elements are most conveniently given in coordinate representation

$$
\left\langle\psi_{p_{A}}^{\dagger}\left|\Pi^{\text {gain }}\right| \psi_{p_{B}}\right\rangle=\int d^{4} r d^{4} r^{\prime} \psi_{p_{A}}^{\dagger(-)}(r) \Pi^{\text {gain }}\left(r, r^{\prime}\right) \psi_{p_{B}}^{(-)}\left(r^{\prime}\right), \text { etc. }
$$

using the current-current correlator (1). In the plane wave limit of identical particles it simply recovers the standard result, cf. [51], valid for penetrating probes

$$
\begin{gathered}
C(q, K)=1+\left[\frac{\left|\int d^{4} r \mathrm{e}^{i q r} \Pi^{\text {gain }}(r, K)\right|^{2}}{\int d^{4} r_{a} \Pi^{\operatorname{gain}}\left(r_{a}, p_{A}\right) \int d^{4} r_{b} \Pi \text { gain }\left(r_{b}, p_{B}\right)}\right]_{p_{A}^{2}=p_{B}^{2}=m^{2}} \\
\text { with } q=p_{A}-p_{B}, \quad K=\frac{1}{2}\left(p_{A}+p_{b}\right) .
\end{gathered}
$$

For the strong coupling case again subtle compensation effect arising between source function and the attenuation along the escape path are expected for the matrix elements (20) in (19). In order to illustrate this we discuss the simplifications emerging again in the semi-classical limit of large particle momenta and for small opening angles between the two detector momenta $\vec{p}_{A}$ and $\vec{p}_{B}$. As Jacobian effects essentially cancel out in the ratio in (20) one can use the eikonal approximation evaluated along straight lines parallel to the averaged momentum $\vec{K}$. Then the wave functions become $\psi_{p_{A}}^{(-)}(r) \approx \mathrm{e}^{i p_{a} r-\frac{1}{2} \chi\left(r, p_{a}\right)}$ etc., where $p_{a}$ and $\chi$ are the local four momentum and the corresponding optical depth. Using further the thermal limit property $\Pi^{\text {gain }}(r, p)=2 p^{0} f_{t h}\left(r, p^{0}\right) \Gamma(r, p)$, the matrix element (20) becomes

$$
\begin{aligned}
\left\langle\psi_{p_{A}}^{\dagger}\left|\Pi^{\text {gain }}\right| \psi_{p_{B}}\right\rangle & \approx \int d^{4} r d^{4} r^{\prime} \Pi^{\text {gain }}\left(r, r^{\prime}\right) \mathrm{e}^{-i p_{a} r+i p_{b} r^{\prime}-\frac{1}{2}\left(\chi\left(r, p_{a}\right)+\chi\left(r^{\prime}, p_{b}\right)\right)} \\
& \approx \int d^{4} \bar{r} d^{4} K 2 K^{0} f_{t h}\left(\bar{r}, K^{0}\right) \Gamma(\bar{r}, K) \mathrm{e}^{i q \bar{r}} \mathrm{e}^{-\chi(\bar{r}, K)}
\end{aligned}
$$

In the last step second order gradients were ignored in defining the averaged optical depth $\chi(\bar{r}, K)$. As a result the coincident yield can be described in some 
quasi-free manner, i.e. using the plane waves form (21), thereby replacing the original source function $\Pi^{\text {gain }}$ by an effective single particle source function

$$
S_{\text {eff }}(r, p)=\Pi^{\text {gain }}(r, p) \mathrm{e}^{-\chi(r, p)},
$$

which accounts for the opaqueness resulting from the corresponding optical depths seen from the detectors.

Alternatively to the analytic results given here one can sample the points of last interaction for the observed particles within transport simulations and this way define the proper emission zone profile in space and time, cf. e.g. [46,55]. In this case the simple yield ratio (21) is still applicable, modulo that one may have to account for the optical deflection of the particles due to the real part of the optical potentials, which maps the detector momenta to the local momenta. This opens the perspective for an imaging analysis of the active source region [56] or region of homogeneity [27,46].

\section{$5 \quad$ Analytic considerations}

The derived continuous decoupling relations raise quite a number of conceptual questions with respect to the observability of structural features of the evolving matter and the standard interpretation in terms of thermal model fits. First question to ask is, under which circumstances fingerprints of the structure of the matter are directly visible by certain probes? As all structural information is contained in the current-current correlation functions, there is a favourable answer: namely by penetrating probes. This has induced worldwide activities to observe photons and lepton pairs resulting from nuclear collisions, since these probes directly see the electromagnetic current-current correlator with its prominent feature to be influenced by vector mesons in the matter.

For all strongly interacting probes, though, the interior is essentially opaque and one is left with surface effects, not only meant spatially but also in the context of time history. Then, however, the structural features are essentially washed out, since the structural parts of the decoupling rates encoded in the damping widths $\Gamma$ essentially integrate to unity. This statement is also true in the near non-equilibrium case, since all non-equilibrium aspects can be encoded in a non-thermal distribution in (9), rather than $f_{\text {th }}$. Thus fingerprints from incoherent decays of resonances with life times less than the decoupling time $\Delta t_{\text {dec }}$ may be less or differently visible.

Dynamically expanding system have one robust feature, namely that on the mean all microscopic damping widths monotonically drop with density, ultimately proportional to the density for elastic scattering processes. This allows simple parametrisations for the time dependence of the microscopic damping 
widths and this way leads to universal (scaling) behaviour between different physical systems.

In the following we consider the emission of slow particles from a spherically expanding uniform fireball or from a Bjorken type expansion [57], once radial flow has built up. The emission is than essentially from a time-like hypersurface. In order to come to simple analytic terms, which display the qualitative features, we assume that the overall volume grows as $V \propto t^{3}$ in time, while the damping rates scale proportional to some power of the density $\rho^{n}$ times an averaged relative velocity. The case $(n=1)$ is essentially relevant for elastic scattering, while $(n=2)$ is more suited for inelastic processes. For simplicity the averaged relative velocity is taken proportional to the square root of the temperature as appropriate for massive particles, i.e.

$$
\begin{aligned}
& V \propto t^{3}, \quad\left\langle v_{r e l}\right\rangle \propto T^{1 / 2} \quad \text { and } \quad \Gamma \propto \rho^{n}\left\langle v_{r e l}\right\rangle \propto t^{-\alpha} \\
& T \propto V^{-(\kappa-1)} \quad \text { and thus } \quad \alpha=3 n+\frac{3}{2}(\kappa-1) .
\end{aligned}
$$

Here we further employed some adiabatic relation between $T$ and $V$ with adiabatic index $\kappa=C_{p} / C_{V}$. These assumptions yield damping rates at the decoupling peak and a corresponding spread in decoupling time, cf. Fig. 1, in the order of

$$
\Gamma\left(t_{\mathrm{dec}}\right)=\alpha \frac{\dot{R}_{\mathrm{dec}}}{R_{\mathrm{dec}}} \quad \text { and } \quad \Delta t_{\mathrm{dec}} \approx \frac{\mathrm{e}^{\alpha /(\alpha-1)}}{\alpha} \frac{R_{\mathrm{dec}}}{\dot{R}_{\mathrm{dec}}}
$$

For the examples presented in Fig. 1 we assumed some typical fireball values,

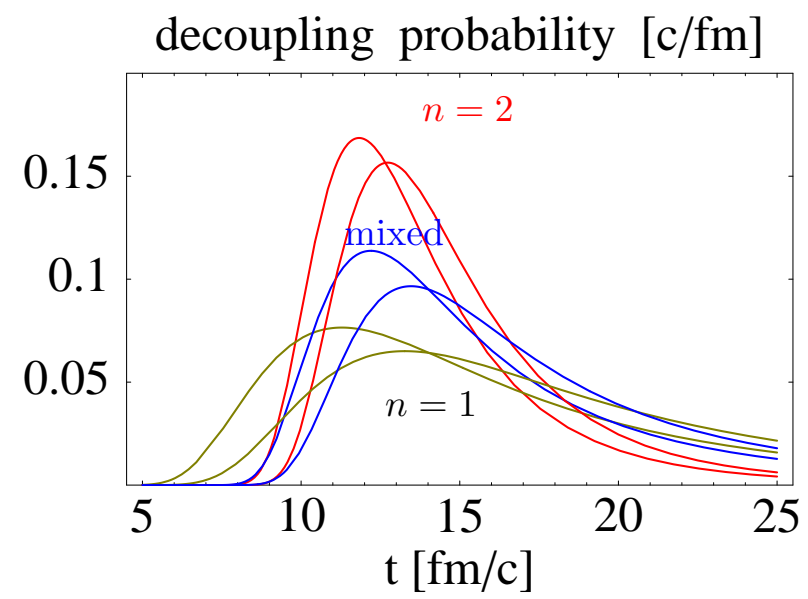

Fig. 1. Decoupling probability $P_{t}(t)$ as a function of time for an expanding homogeneous fireball for the three decoupling scenarios, where the rate depends linear or quadratically on the density $(n=1,2)$ or a mixed scenario as discussed in the text. The three curves slightly displaced to the right result from scaling all rates by a factor 1.5 . 
namely a freeze-out radius of $R_{\mathrm{dec}} \approx 6 \mathrm{fm}$ and a collective velocity $\dot{R}_{\mathrm{dec}}=0.5 \mathrm{c}$ for the nuclear collision case. Through (27) these collective parameters already determine the damping values at the decoupling peak, namely $\Gamma_{\mathrm{dec}}=0.5 \mathrm{c} / \mathrm{fm}$ $\approx 100 \mathrm{MeV}$ for inelastic processes $(n=2)$ and still $50 \mathrm{MeV}$ for scattering processes $(n=1)$. Beside these two options a mixed case with assumed $30 \%$ of $n=1$ admixture at decoupling time $t_{\mathrm{dec}}=12 \mathrm{fm} / \mathrm{c}$ might be appropriate for the kinetic or thermal decoupling, since in this case both, chemical and scattering processes, do contribute. Also here $\Gamma_{\text {dec }}$ emerges to about $100 \mathrm{MeV}$. Microscopically, of course, it is the time behaviour of $\Gamma(t)$ that determines the decoupling conditions.

The here obtained decoupling durations $\Delta t_{\text {dec }}$ lie above $5 \mathrm{fm} / \mathrm{c}$, during which, as a more universal result, the volume almost increases by an order of magnitude. The robust microscopic figures in this respect, however, is the variation of the damping width between start (i) and stop (f) of the decoupling process (taken at full width half maximum). They vary by about a factor $\mathrm{e}^{-\mathrm{e}} \approx 1 / 15$, i.e. $\Gamma_{\mathrm{i}}: \Gamma_{\mathrm{dec}}: \Gamma_{\mathrm{f}} \approx \mathrm{e}^{\frac{1}{2} \mathrm{e}}: 1: \mathrm{e}^{-\frac{1}{2} \mathrm{e}} \approx 390 \mathrm{MeV}: 100 \mathrm{MeV}: 26 \mathrm{MeV}$. The latter values of $\Gamma$ representative for the above cases by themselves are tremendous news: during the entire decoupling process the resulting decoupling rates (or damping widths) are significant and of same order or even larger than the mean kinetic energies of the constituents.

In order to see the sensitivity on the absolute damping strength a second set of curves is supplemented in Fig. 1, where all damping rates are scaled up by $50 \%$, correspondingly leading to slightly later decouplings. The corresponding curves scale to one another, if plotted in dimensionless variables and thus equally apply to other physical processes like e.g. the photon decoupling during the early universe. The latter is reported [45] to occur in a time window with the cosmological red shift factor varying between $Z=1300$ and 800 with peak at $Z \approx 1050$, well in line with the time profiles deduced here. In the early universe case, however, the implied temperature drop during decoupling is of minutes harm for the nowadays observed cosmological microwave background radiation $(\mathrm{CMB})$, since it is perfectly accompanied and exactly compensated by the cosmological red shift. Thus fluctuations in the CMB temperatures observed in different celestial directions result as tiny as on the $10^{-5}$ level.

For the nuclear collision case there is a priory no such compensation to be expected. Rather from the above results one expects a significant spread in the thermodynamic conditions during the continuous decoupling or freeze-out. The time profiles of Fig. 1 can accordingly be transformed to the resulting probability distribution in temperature via

$$
P_{T}(T)=P_{t}(t(T)) \frac{d t}{d T}
$$

where $T(t)$ and its inverse $t(T)$ describe the temperature change with time due 

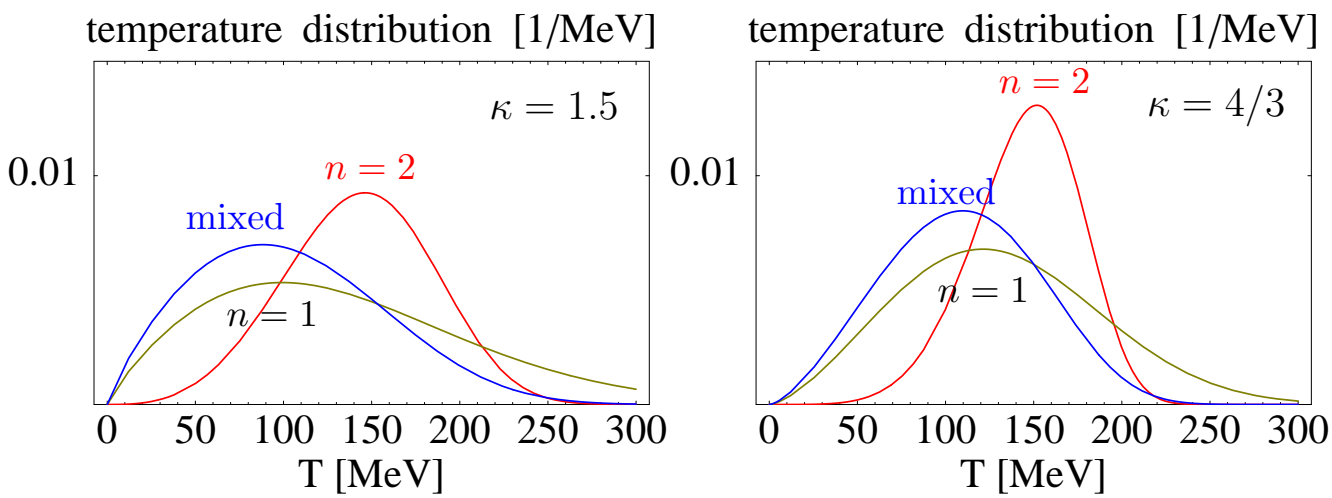

Fig. 2. Temperature distributions $P_{T}(T)$ resulting from the three different decoupling scenarios shown in Fig. 1. Left and right panels for EoS with adiabatic ratios $\kappa=1.5$ and $4 / 3$, respectively.

to the underlying equation of states (EoS). For two simple example EoS with adiabatic ratios $\kappa=C_{p} / C_{V}=1.5$ and $4 / 3$, respectively the corresponding distribution in $T$ are shown in the two panels of Fig. 2. While $\kappa=4 / 3$ corresponds to an ideal gas of massless particles, e.g. photons in the early universe, the former interpolates between this massless case and the fully massive ideal gas case $(M \gg T)$ for which $\kappa=5 / 3$. For orientation we used a temperature value of $T_{\mathrm{dec}}=160 \mathrm{MeV}$ at the decoupling peak around $t_{\mathrm{dec}}=12 \mathrm{fm} / \mathrm{c}$. One also notices that due to this transformation one does not only obtain sizable spreads in the temperature distributions, but also that the peaks in $T$ may significantly be shifted downwards compared to the decoupling value of $T_{\text {dec }}=160 \mathrm{MeV}$. Thus peak positions in $T$ differ from those in $t$, illustrating that depending on the observable the optimal decoupling condition may change because of the finite duration of the decoupling.

Since the coupling widths $\Gamma$ significantly change with volume and temperature, and hence drop in time, their absolute value at some given thermodynamic condition is less important than the relative speed by which these rates drop. This can be seen by comparing the results shown in both figures for the $n=$ 1 or also the "mixed" scenario, which have more a moderate drop of the coupling, relative to the faster drop for $n=2$. The resulting distributions in temperature are vastly different. One obtains a much more narrow distribution peaking slightly below the anticipated decoupling $T_{\mathrm{dec}}$ of $160 \mathrm{MeV}$ for the fast decoupling, while the mixed scenario relevant for kinetic decoupling peaks at much lower $T$ with much broader width.

Plots similar to the ones in Fig. 1 were obtained already in various transport calculations like in [2] for kaon production at SIS, or in $[3,4]$ for the production of mesons and baryons at the CERN SPS, or in [5] for collisions at RHIC with comparable or even longer freeze-out durations. Still, those results did not quite trigger an alert towards a critical reinspection of the ubiquitous application of instantaneous freeze-out concepts. 


\section{Decoupling phenomena}

In the following we discuss several physical phenomena in the light of the long decoupling times.

\section{Chemical Freeze-out}

Over years nuclear collision data taken at the Brookhaven AGS, the CERN SPS accelerators and the RHIC collider were analysed in terms of thermal freeze-out models. In particular the relative abundances of the various particle species delivered the conditions on the chemical freeze-out in terms of temperature $T_{\text {chem }}$ and baryon chemical potential $\mu_{B}$. These fits provided data based freeze-out marks in a QCD phase diagram of $T$ versus $\mu_{B}$ [11-13]. Thereby the $T_{\text {chem }}$ did not only saturate with increasing collision energy at a value around $165 \mathrm{MeV}$. For SPS and RHIC events good $\chi$-squared fits lead to relatively sharply determined freeze-out temperatures $T_{\text {chem }}$ for each collision energy, despite the here diagnosed freeze-out durations, during which the overall volume increases by almost an order of magnitude. For ordinary EoS $T$ steadily drops with increasing volume and one would expect a resulting spread in $T$ similar to those given in Fig. 2. If the narrow temperature fits consolidate (see however [58]), could it be a sign, that the EoS is not such trivial and indeed contains ingredients which provide a halt in the temperature drop during chemical decoupling? This would be an interesting chance, that results from the considerations given here. Phase transitions with considerable latent-heat release, such as the QCD to hadron gas phase transition, may be a possible explanation in this respect.

\section{Phase transition scenarios}

Phase transition involve a restructuring of the matter. The corresponding transition rates and durations essentially obey similar uncertainty relations as Eq. (12) $\Gamma_{\text {trans }} \Delta t_{\text {trans }} \approx$ e. Alongside e.g. the deconfinement-confinement transition is accompanied by a strong reduction of the entropy density, implying a considerable amount of latent heat released during the transition. Thereby for the discussion given here it is less important, whether the transition is of first or second order or even cross over, a distinction of less importance for finite systems during a finite transition time. Important is that across a narrow window in temperature a certain amount of latent heat is released. Such transitions then lead to a sensitive slowing down in the temperature drop during the transition process, as e.g. shown in a chemical rate equation model suggested for the description of the QGP to the hadron phase transition $[6,7]$. 
Irrespective of the specific assumptions employed, on thermo- and transportdynamically sound grounds this model provided qualitative insight into the transition dynamics. The dynamical investigations within a first order phasetransition scenario [59] show that such transitions take a considerable amount of time (about 6 - $10 \mathrm{fm} / \mathrm{c}$ depending on system size and beam energy). During this transition the temperature is essentially kept constant due to the release of latent heat, much like if coupled to a thermostat, while the volume increases by one order of magnitude though, cf. Fig. 1 in [6]. In such transitions the rates governing the abundances of the species in both phases are highly non-linear with density or temperature $[6,60]$. In order to provide the proper equilibrium phase they have to be governed by corresponding driving potential, such as the chemical potentials of the species resulting from the underlying EoS. Such scenarios will indeed provide much more narrow distributions in the resulting decoupling temperatures for the chemical abundances than in the simple EoS cases discussed here, cf. Fig.2, since one expects the chemical decoupling to happen right after the confinement transition.

\section{Thermal Freeze-out}

For the kinetic or thermal freeze-out several competing effects enter, such as thermal motion versus flow, latent heat effects and the influence of the optical potential.

Quite some compensation occurs between the thermal random motion and the collective flow that builds up during the expansion, cf. the results from an exactly solvable model [27]. An early freeze-out with small flow and a large temperature and a later freeze-out with larger flow and smaller temperature essentially lead to the same observed momentum spectra of the particles. Thus nearly invisible for a single component, flow effects can be isolated in comparing the spectra of species with different masses, cf. e.g. the analysis in [61]. Thereby the fits are not unique and therefore do not immediately contradict with a wider spread in kinetic decoupling temperatures.

The special effect of the role of the escape probability (4) can be studied in comparing the freeze-out time structure of weakly and strongly coupling probes of similar or even identical mass. While the weakly interacting probes are essentially created during the entire collision process with an enhanced production during the initial phase for subthreshold processes, for the strongly interacting probes the freeze-out phase is shifted to the very late stage of the reaction. Transport investigations for the subthreshold production of $\mathrm{K}^{+}$ (weak) to $K^{-}$(strong) in [2], or comparing the $\Omega^{-}$(weak) to proton (strong) production in [3] nicely confirm the here developed pictures with freeze-out durations that are well in agreement with the presented behaviour in the context of Fig. 1. That even absorption processes of rare probes can be counter- 
balanced by rare multi-particle production processes was substantiated e.g. for anti-baryon production yields $[62,63]$.

Finger prints of short lived resonances

With the freeze-out duration a new time scale enters, which divides resonances into long and short lived ones. While long lived resonances survive the decoupling with their vacuum spectral function and subsequent vacuum decay patters, resonances with comparable or much shorter live times are affected by the decoupling dynamics. The question then arises, which possible signals do survive the decoupling process, e.g. for pions created through the decay of the $\Delta$-resonance. Interesting in this context are the Coulomb corrected $\pi^{+}$ and $\pi^{-}$spectra [64] and $\pi^{0}$ data [65] taken at the GSI SIS. They show a two slope behaviour with a steeper rise towards small pion energies. Explanations, which attributed this low energy enhancement to the incoherent decay of the Delta resonance, are disfavoured in view of relation (10), since the damping dependent factors integrate to unity across the escape path leaving the opaque zone. The physical origin of this is, that the visible layers are different in depth, such that the enhanced resonance production is essentially compensated by the corresponding reduced mean free path. The net result ultimately leads to Planck's law of black-body radiation in the opaque limit. Yet, such resonances do influence the corresponding optical potential of the emitted particle as encoded in $\Pi^{\mathrm{R}}$. In lowest order virial expansion the latter is determined by the corresponding scattering phase-shifts [66]. For the $(\pi, N, \Delta)$ system this contribution to the optical potential leads to some steepening of the pion spectra at lower c.m. energies $[67,68]$.

Composite-particle formation

Composite-particle formation processes at freeze-out can be endo- or exothermic. The formation of composites, such as deuterons or heavier nuclei for example, freezes degrees of freedom, thus releasing latent heat, that has then to be recoupled to the source according to Eqs. (8). Except for chemical freeze-out models this recoupling is mostly not accounted for e.g. in coalescence models. A microscopic description of composite light nuclei formation was formulated in [69] and further developed in [70]. Thereby composite particles are dynamically formed by multi-particle processes which respect the conservation laws.

A particular example case of endo- and exothermic emission was studied in a conserving surface evaporation model applied to the decay of a thermal quark-gluon plasma glob [71]. While the emission of pions cooled the source, the emission of baryons simultaneously respecting energy and baryon number conservation indeed heated the source for the employed EoS. An interesting 
feature in the context addressed in this note is, that this combined action lead to a stabilised source temperature during emission, much like for azeotropic distillation processes.

\section{Interferometry imaging and the HBT puzzle}

Prior to the RHIC experiments a wealth of model calculations appeared, cf. the reviews $[53,54]$, that predicted large values for the ratio of two special HBT "radii" $R_{\text {out }} / R_{\text {side }}[72]$ with values beyond 4 or even much larger. However the finally measured data $[73,74]$ arrived at values, which are similar to those already found at the CERN SPS, cf. [75-77], namely between 0.9 and 1.2. The origin of this puzzle [78] had many reasons. It partly resided in the use of simple freeze-out concepts, some times in conjunction with an inappropriate HBT recipe for the source's mean square lifetime, namely $\left\langle\left(v_{x} t\right)^{2}\right\rangle \approx R_{\text {out }}^{2}-R_{\text {side }}^{2}$. As it ignores space-time correlations ( $v_{x}$ denotes the particles' velocity in out direction), this relation is valid only under severe restrictions, which prevent its usage for strongly interacting probes, see the discussion in [51], around Eq. (3.22). Besides space-momentum and space-time correlations already built up by the source encoded in $\Pi^{\text {gain }}$, further correlation arise from the here discussed restricted optical view due to the final state damping as included in $S_{\text {eff }}$, cf. (24). Together with a kind of collective Hubble expansion of the source $[46,53,55,79]$ strong positive $x$ - $t$ correlations are built up, which significantly reduce $R_{\text {out }}$ despite the long freeze-out times, since [80]

$$
R_{\text {out }}^{2}=\left\langle\left(x-v_{x} t\right)^{2}\right\rangle-\left\langle\left(x-v_{x} t\right)\right\rangle^{2}, \quad \text { while } R_{\text {side }}^{2}=\left\langle y^{2}\right\rangle,
$$

with spatial coordinates $x$ and $y$ pointing in out and side direction. The above averages are meant to be taken in the sense of Eq. (23). Thereby the optical attenuation included in $S_{\text {eff }}$ leads to a possible breaking of some of the symmetries inherent in the original source distribution $\Pi^{\text {gain }}$ and to corresponding shifts in the centre of gravity of $S_{\text {eff }}$ relative to that of $\Pi^{\text {gain }}$. The here discussed considerations are nicely confirmed by recent hybrid transport simulations that appeared during the revision stage of this paper $[46,55,79]$. These model calculations did not only find the here advocated long freeze-out durations (above $10 \mathrm{fm} / \mathrm{c}$ ). At the same time they provided a solution to the HBT puzzle, as the events emerged compatible with $R_{\text {out }} / R_{\text {side }}$ close to unity.

In order to account for the distortion effects a technically involved distorted wave formalism was developed in Ref. [21] and applied to the analysis of pion coincidence data at RHIC. This approach however, did not quite respect the tight relation between the properties of the source $\Pi^{\text {gain }}$ and the corresponding optical potential $\Pi^{\mathrm{R}}$ determining the wave functions. Both ingredients were parametrised independently, although they are to be obtained from the same current-current correlation function as shown here. The absorption effects can 
even be considered in a simpler way through the semi-classical effective source function $S_{\text {eff }}$, cf. (24), to be used in the plane wave formula (21).

\section{Concluding remarks}

From general perspectives decoupling processes in dynamically expanding systems show quite a universal behaviour. They proceed during a considerable time span, during which the damping rates drop by more than an order of magnitude implying alongside a sizable increase of the system's volume. The here formulated microscopic relations generalise instantaneous recipes and provide a conserving scheme for transition and decoupling processes in compliance with transport- and thermodynamic constraints such as detailed balance, unitarity and entropy requirements. Although the physical results are generally obtainable in much more detail from numerical transport simulations, the analytic formulae given here present a didactic view on such processes. This includes simple pocket formulae, which among others provide a kind of uncertainty relation between the damping rate of the decoupling particle at the decoupling peak and the decoupling duration. For the decoupling of strongly interacting probes thereby a subtle compensation effect emerges which leads to a generic behaviour. Like for Planck's radiation law it essentially wipes out the memory on the microscopic structural properties of the source. Despite the complications due to the finite decoupling time the presented scheme opens the perspective for improved future treatments of fluid dynamic calculations.

For applications to nuclear collisions the here diagnosed long decoupling and freeze-out times are both a challenge but also a chance: a chance to map out the thermodynamic properties of the expanding collision zone during the freeze-out of various probes. An observation of a quite narrow distribution in temperature, for example, could point towards effects that significantly slow down the temperature drop during expansion, and this way provide hints towards the underlying equation of state or possible phase transition effects. Therefore this note is meant as a stimulation to reconsider the analyses of nuclear collisions data in the light of the results and discussions given here. Promising steps towards this goal were recently achieved by hybrid model calculations $[46,55]$, where the entire decoupling stage is treated within kinetic transport.

\section{Acknowledgement}

The author acknowledges encouraging and clarifying discussions with A. Andronic, P. Braun-Munzinger, P. Danielewicz, B. Friman, M. Gyulassy, Yu.B. Ivanov, E. Kolomeitsev, S. Leupold, H. Oeschler, S. Pratt, K. Redlich and D.N. Voskresensky. 


\section{References}

[1] J. W. Harris, B. Müller, Ann. Rev. Nucl. Part. Sci. 46 (1996) 71-107.

[2] C. Hartnack, H. Oeschler, J. Aichelin, arXiv:0712.0316 [nucl-th].

[3] H. van Hecke, H. Sorge, N. Xu, Phys. Rev. Lett. 81 (1998) 5764-5767.

[4] S. A. Bass, et al., Prog. Part. Nucl. Phys. 42 (1999) 313-322.

[5] C. Nonaka, S. A. Bass, Phys. Rev. C75 (2007) 014902.

[6] H.-W. Barz, B. L. Friman, J. Knoll, H. Schulz, Nucl. Phys. A 484 (1988) 661.

[7] H.-W. Barz, B. L. Friman, J. Knoll, H. Schulz, Nucl. Phys. A 519 (1990) 831.

[8] H. Sato, K. Yazaki, Phys. Lett. B98 (1981) 153-157.

[9] T. S. Biro, P. Levai, J. Zimanyi, Phys. Rev. C59 (1999) 1574-1584.

[10] F. Cooper, G. Frye, Phys. Rev. D 10 (1974) 186.

[11] P. Braun-Munzinger, J. Stachel, Nucl. Phys. A606 (1996) 320-328.

[12] P. Braun-Munzinger, J. Stachel, Nucl. Phys. A638 (1998) 3-18.

[13] J. Cleymans, K. Redlich, Phys. Rev. Lett. 81 (1998) 5284-5286.

[14] A. Andronic, P. Braun-Munzinger, J. Stachel, Nucl. Phys. A772 (2006) 167-199.

[15] L. P. Kadanoff, G. Baym, Quantum Statistical Mechanics, Benjamin, 1962.

[16] J. Knoll, to be published.

[17] M. Gyulassy, S. K. Kauffmann, L. W. Wilson, Phys. Rev. C20 (1979) 2267-2292.

[18] P. Danielewicz, P. Schuck, Phys. Lett. B 274 (1992) 268-274.

[19] R. Hanbury Brown, R. Q. Twiss, Nature 178 (1956) 1046-1048.

[20] H. W. Barz, Phys. Rev. C59 (1999) 2214-2220.

[21] G. A. Miller, J. G. Cramer, J. Phys. G34 (2007) 703-740.

[22] F. Grassi, Y. Hama, T. Kodama, Phys. Lett. B355 (1995) 9-14.

[23] F. Grassi, Y. Hama, T. Kodama, Z. Phys. C73 (1996) 153-160.

[24] L. P. Csernai, Z. Lazar, D. Molnar, Heavy Ion Phys. 5 (1997) 467-474.

[25] V. K. Magas, et al., Heavy Ion Phys. 9 (1999) 193-216.

[26] E. Molnar, et al., Phys. Rev. C74 (2006) 024907.

[27] Y. M. Sinyukov, S. V. Akkelin, Y. Hama, Phys. Rev. Lett. 89 (2002) 052301.

[28] A. Messiah, Quantum Mechanics, Vol. II, North-Holland Pub. Com., 1969.

[29] R. G. Newton, Scattering Theory of Waves and Particles, Springer, New York, USA, 1982.

[30] R. Courant, D. Hilbert, Methoden der Mathematischen Physik, Vol. II, Springer, 1968.

[31] Y. B. Ivanov, J. Knoll, D. N. Voskresensky, Nucl. Phys. A 672 (2000) 313.

[32] J. Knoll, Y. B. Ivanov, D. N. Voskresensky, Ann. Phys.(NY) 293 (2001) 126.

[33] S. Leupold, Nucl. Phys. A 672 (2000) 475.

[34] W. Cassing, S. Juchem, Nucl. Phys. A 672 (2000) 417.

[35] J. H. van Vleck, Proc. Nat. Acad. Sci. 14 (1928) 178.

[36] J. Knoll, D. N. Voskresensky, Annals Phys. 249 (1996) 532-581.

[37] U. Katscher, et al., Z. Phys. A346 (1993) 209-216.

[38] J. Brachmann, et al., Nucl. Phys. A619 (1997) 391-412.

[39] Y. B. Ivanov, V. N. Ruuskikh, V. D. Toneev, Phys. Rev. C 73 (2006) 44904. 
[40] V. D. Toneev, Y. B. Ivanov, E. G. Nikonov, W. Norenberg, V. N. Russkikh, Phys. Part. Nucl. Lett. 2 (2005) 288.

[41] V. N. Russkikh, Y. B. Ivanov, E. G. Nikonov, W. Norenberg, V. D. Toneev, Phys. Atom. Nucl. 67 (2004) 199.

[42] V. N. Russkikh, Y. B. Ivanov, Phys. Rev. C 76 (2007) 054907.

[43] J. Knoll, R. Schaeffer, Annals Phys. 97 (1976) 307-366.

[44] U. Seljak, M. Zaldarriaga, Astrophys. J. 469 (1996) 437-444.

[45] V. Mukhanov, Physical Foundation of Cosmology, Cambridge University Press, 2005.

[46] S. V. Akkelin, Y. Hama, I. A. Karpenko, Y. M. Sinyukov, Phys. Rev. C78 (2008) 034906.

[47] K. A. Bugaev, Nucl. Phys. A606 (1996) 559-567.

[48] K. A. Bugaev, M. I. Gorenstein, W. Greiner, J. Phys. G25 (1999) 2147-2160.

[49] J. J. Neumann, B. Lavrenchuk, G. I. Fai, Heavy Ion Phys. 5 (1997) 27.

[50] H. van Hees, R. Rapp, Nucl. Phys. A806 (2008) 339.

[51] U. A. Wiedemann, U. W. Heinz, Phys. Rept. 319 (1999) 145-230.

[52] M. A. Lisa, S. Pratt, R. Soltz, U. Wiedemann, Ann. Rev. Nucl. Part. Sci. 55 (2005) 357-402.

[53] T. Csörgö, J. Phys. Conf. Ser. 50 (2006) 259-270.

[54] S. S. Padula, Braz. J. Phys. 35 (2005) 70-99.

[55] S. Pratt, J. Vredevoogd, arXiv:0809.0516 [nucl-th].

[56] D. A. Brown, P. Danielewicz, Phys. Lett. B398 (1997) 252-258.

[57] J. D. Bjorken, Phys. Rev. D27 (1983) 140-151.

[58] A. Dumitru, L. Portugal, D. Zschiesche, Phys. Rev. C73 (2006) 024902.

[59] H. W. Barz, B. L. Friman, J. Knoll, H. Schulz, Phys. Rev. D40 (1989) 157.

[60] P. Braun-Munzinger, J. Stachel, C. Wetterich, Phys. Lett. B596 (2004) 61-69.

[61] W. Reisdorf, et al. [FOPI Collaboration], Nucl. Phys. A612 (1997) 493-556.

[62] R. Rapp, E. V. Shuryak, Phys. Rev. Lett. 86 (2001) 2980-2983.

[63] C. Greiner, S. Leupold, J. Phys. G27 (2001) L95-L102.

[64] B. Hong, et al. [FOPI Collaboration], Phys. Rev. C57 (1998) 244-253.

[65] O. Schwalb, et al., Phys. Lett. B321 (1994) 20-25.

[66] E. Beth, G. E. Uhlenbeck, Physica 4 (1937) 915.

[67] W. Weinhold, B. Friman, W. Nörenberg, Phys. Lett. B 433 (1998) 236.

[68] Y. B. Ivanov, J. Knoll, D. N. Voskresensky, Nucl. Phys. A 657 (1999) 413.

[69] E. A. Remler, Ann. Phys. 136 (1981) 293.

[70] P. Danielewicz, G. F. Bertsch, Nucl. Phys. A 533 (1991) 712.

[71] H. W. Barz, B. L. Friman, J. Knoll, H. Schulz, Phys. Lett. B242 (1990) 328-333.

[72] G. F. Bertsch, Nucl. Phys. A498 (1989) 173c-180c.

[73] C. Adler, et al., Phys. Rev. Lett. 87 (2001) 082301.

[74] S. S. Adler, et al., Phys. Rev. Lett. 93 (2004) 152302.

[75] J. Baechler, et al., Nucl. Phys. A525 (1991) 327c-332c.

[76] T. Alber, Nucl. Phys. A590 (1995) 453c-458c.

[77] D. Adamova, et al., Nucl. Phys. A714 (2003) 124-144.

[78] M. Gyulassy, Lect. Notes Phys. 583 (2002) 37-79.

[79] S. Pratt, arXiv:0811.3863 [nucl-th].

[80] M. Herrmann, G. F. Bertsch, Phys. Rev. C51 (1995) 328-338. 